\title{
Spatial Cognition and Neuro-Mimetic Navigation: A Model of Hippocampal Place Cell Activity
}

\author{
Angelo Arleo and Wulfram Gerstner \\ Centre for Neuro-Mimetic Systems, MANTRA, \\ Swiss Federal Institute of Technology Lausanne, \\ CH-1015, Lausanne, EPFL, Switzerland.
}

Received: 02 July 1999 / Accepted in revised form: 20 March 2000

\begin{abstract}
A computational model of hippocampal activity during spatial cognition and navigation tasks is presented. The spatial representation in our model of the rat hippocampus is built on-line during exploration via two processing streams. An allothetic vision-based representation is built by unsupervised Hebbian learning extracting spatio-temporal properties of the environment from visual input. An idiothetic representation is learned based on internal movement-related information provided by path integration. On the level of the hippocampus, allothetic and idiothetic representations are integrated to yield a stable representation of the environment by a population of localized overlapping CA3-CA1 place fields. The hippocampal spatial representation is used as a basis for goal-oriented spatial behavior. We focus on the neural pathway connecting the hippocampus to the nucleus accumbens. Place cells drive a population of locomotor action neurons in the nucleus accumbens. Reward-based learning is applied to map place cell activity into action cell activity. The ensemble action cell activity provides navigational maps to support spatial behavior. We present experimental results obtained with a mobile Khepera robot.
\end{abstract}

\section{Introduction}

As the complexity of the tasks and the perceptual capabilities of biological organisms increase, an explicit spatial representation of the environment appears to be employed as a cognitive basis to support navigation [25]. In rodents, hippocampal place cells exhibit such a spatial representation property. Recordings from single place cells in the rat hippocampus $[24,25]$ show that these neurons fire as a function of the rat's spatial location. A place cell shows action potentials only when the animal is in a specific region of the environment, which defines the place field of the cell. Place cells have been observed in the hippocampus proper (CA3 and CA1 pyramidal cells) [24, 41], and in other extra-hippocampal areas such as the dentate gyrus [16], the entorhinal cortex [29], the subiculum [36], and the parasubiculum [38].

Correspondence to: Angelo Arleo, angelo.arleo@epfl.ch
In addition, recent experimental findings show the existence of head-direction cells, neurons whose activity is tuned to the orientation of the rat's head in the azimuthal plane. Each head-direction cell fires maximally when the rat's head is oriented in a specific direction, regardless of the orientation of the head with respect to the body, and of the rat's spatial location. Thus, the ensemble activity of head-direction cells provides a neural allocentric compass. Head-direction cells have been observed in the hippocampal formation and in particular in the postsubiculum [39], in the anterior thalamic nuclei $[1,17]$, and in the lateral mammillary nuclei $[18]$.

Place coding and directional sense are crucial for solving spatial learning tasks. Hippocampal lesions seriously impair the rat's performance in spatial tasks (see [31] for an experimental review). This supports the hypothesis that the hippocampus plays a functional role in rodent navigation, and that it provides a neural basis for spatial cognition and spatial behavior [24, 25, 41, 20].

Hippocampal place fields are determined by a combination of environmental cues whose mutual relationships code for the current animal location [25]. Experiments on rats show that visual cues are of eminent importance for the formation of place fields [17]. Nevertheless, rats also rely on other allothetic non-visual stimuli, such as auditory, olfactory, and somatosensory cues [15]. Moreover, place cells can maintain stable receptive fields even in absence of reliable allothetic cues (e.g., in the dark) [28]. This suggests a complex architecture where multimodal sensory information is used for learning and maintaining hippocampal place fields. In the dark, for instance, idiothetic information (e.g., proprioceptive and vestibular stimuli) might partially replace external cues [10].

We present a computational model of the hippocampus which relies on the idea of sensor-fusion to drive place cell activity. External cues and internal self-generated information are integrated for establishing and maintaining hippocampal place fields. Receptive fields are learned by extracting spatio-temporal properties of the environment. Incoming visual stimuli are interpreted by means of neurons that only respond to combinations of specific visual patterns. The activity of these neu- 
rons implicitly represents properties like agent-landmark distance and egocentric orientation to visual cues. In a further step, the activity of several of these neurons is combined to yield place cell activity. Unsupervised Hebbian learning is used to build the hippocampal neural structure incrementally. In addition to visual input we also consider idiothetic information. An extrahippocampal path integrator drives Gaussian-tuned neurons modeling internal movement-related stimuli. During the agent-environment interaction, synapses between visually driven cells and path-integration neurons are established by means of Hebbian learning. This allows us to correlate allothetic and idiothetic cues to drive place cell activity. The proposed model results in a neural spatial representation consisting of a population of localized overlapping place fields (modeling the activity of CA1 and CA3 pyramidal cells). To interpret the ensemble place cell activity as spatial location we apply a population vector coding scheme [14, 41].

In order to accomplish its functional role in spatial behavior, the proposed hippocampal model must incorporate the knowledge about relationships between the environment, its obstacles and specific target locations. As in Brown and Sharp [2], and in Burgess et al. [4], we apply reinforcement learning [37] to enable targetoriented navigation based on hippocampal place cell activity. We focus on a specific neural pathway, namely the fornix projection, connecting the hippocampus (in particular the CA1 region) to the nucleus accumbens. The latter is an extra-hippocampal structure that is probably involved in reward-based goal memory and in locomotor behavior [2, 31]. Place cell activity drives a population of locomotor action neurons in the nucleus accumbens [2]. Synaptic efficacy between CA1 cells and action cells is changed as a function of target-related reward signals. This results in an ensemble activity of the action neurons that provides a navigational map to support spatial behavior.

In order to evaluate our hippocampal model in a real context, we have implemented it on a Khepera miniature mobile robot (Fig. 6(b)). Allothetic information is provided by a linear vision system, consisting of 64 photo-receptors covering 36 degrees of azimuthal range. Eight infrared sensors provide obstacle detection capability (similar to whiskers). Internal movement-related information is provided by dead-reckoning (odometry). Robotics offers a useful tool to validate models of functionalities in neuro-physiological processes [27]. Artificial agents are simpler and more experimentally transparent than biological systems, which makes them appealing for understanding the nature of the underlying mechanisms of animal behavior.

Our approach is similar in spirit to earlier studies $[4,32,31,13,40,19,2]$. In contrast to Burgess, Recce and O'Keefe [4], we do not directly use metric information (i.e., distance to visual cues) as input for the model. Rather, we interpret visual properties by learning a population of neurons sensitive to specific visual stimulation. Moreover, there is no path integration in the model of Burgess et al.. In contrast with their model, we consider, along with vision, the path integrator as an important constituent of our hippocampal model. This allows us to
account for the existence of place fields in the absence of visual cues (e.g., in complete darkness) [28]. Redish and Touretzky $[32,31]$ have put forward a comprehensive theory of the hippocampal functionality where place fields are important ingredients. Our approach puts the focus on how place fields in the CA3-CA1 areas might be built from multimodal sensory inputs (i.e., vision and path integration). Gaussier et al. [13] propose a model of the hippocampal functionality in long-term consolidation and temporal sequence processing. Trullier and Meyer [40] build a topological representation of the environment from sequences of local views. In contrast to those two models, temporal aspects are, in our approach, mainly implicit in the path integration. In contrast to Mallot et al. [19], who construct a sparse topological representation, our representation is rather redundant and uses a large number of place cells. Similarly to Brown and Sharp [2], we consider the cell activity in the nucleus accumbens to guide navigation. However, we do not propose an explicit model for the nucleus accumbens. Finally, similarly to Schultz, Dayan and Montague [35, 6] we consider the role of dopaminergic neurons in rewardbased learning. However, we study hippocampal goaloriented navigation in a real agent-environment context.

\section{Spatial Representation in the Hippocampus}

\subsection{Biological Background}

Fig. 1 shows the functional rationale behind the model: (i) External stimuli (i.e., visual data) are interpreted to characterize distinct regions of the environment by distinct sensory configurations. This results in an allothetic (vision-based) spatial representation consistent with the local view hypothesis suggested by McNaughton in 1989 [20]. (ii) Internal movement-related stimuli (i.e., proprioceptive and vestibular) are integrated over time to provide an idiothetic (path integration-based) representation. (iii) Allothetic and idiothetic representations are combined to form a stable spatial representation in the hippocampus (CA3-CA1 place fields). (iv) Spatial navigation is achieved based on place cell activity, desired targets, and rewarding stimulation.

Fig. 2 shows the anatomical framework underlying our computational model. The hippocampus proper (Cshaped structure in Fig. 2) consists of the CA3-CA1 areas. The hippocampal formation consists of the hippocampus proper, the dentate gyrus (DG), the entorhinal cortex (in particular, we consider superficial (sEC) and medial (mEC) entorhinal regions), and the subicu$\operatorname{lum}(\mathrm{SC})$.

The hippocampus receives multimodal highly processed sensory information mainly from neocortical areas, and from subcortical areas (e.g., inputs from the medial septum via the fornix fiber bundle) [3]. We focus on neocortical inputs and in particular on the information coming from the posterior parietal cortex. Lesion data on humans and monkeys, suggest that parietal areas are involved in spatial cognition and spatial behavior 


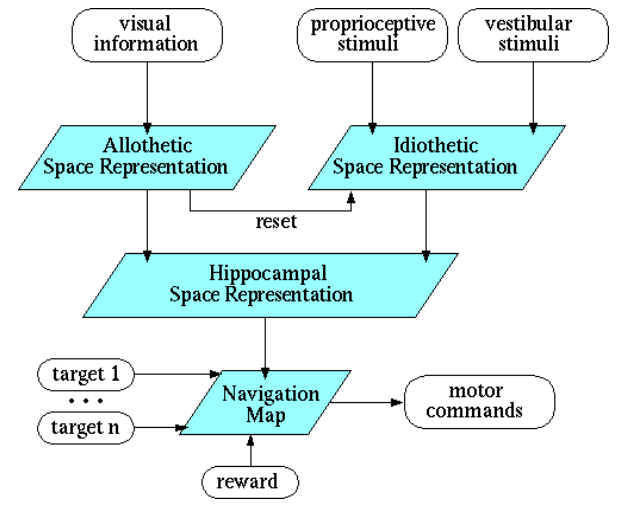

Fig. 1. Functional overview of the model. Allothetic and idiothetic stimuli are combined to yield the hippocampal space representation. Navigation is based on place cell activity, desired targets, and rewards.

[3]. The posterior parietal cortex receives inputs from visual, sensory-motor, and somatosensory cortices. This information reaches the entorhinal regions, within the hippocampal formation, via the parahippocampal (PaHi) and the perirhinal $(\mathrm{PeRh})$ cortices. Finally, the entorhinal cortex projects to the hippocampus proper via the perforant path (PP) [3].

As previously mentioned, we consider the spatial representation in the CA3-CA1 areas as the result of integrating idiothetic and allothetic representations (Fig. 1). The idiothetic representation is assumed to be environment-independent. Recordings from cells in the medial entorhinal cortex $(\mathrm{mEC})$ show place fields with a topology-preserving property across different environments $[29,31]$. Thus, we suppose that the idiothetic representation takes place in the medial entorhinal cortex. A fundamental contribution to build the idiothetic space representation in $\mathrm{mEC}$ comes from the head-direction system (Fig. 2). The latter is formed by the neural circuit including the lateral mammillary nuclei (LMN), the anterodorsal nucleus of anterior thalamus (ADN), and the postsubiculum (poS) $[1,31]$. Head-direction information is projected to the medial entorhinal cortex $(\mathrm{mEC})$ from the postsubiculum (poS).

On the other hand, we suppose that the allothetic representation is formed in the superficial entorhinal cortex (sEC) [31]. Superficial layers of the entorhinal cortex receive spatial information about allothetic landmarks (local view) from the posterior parietal cortex, and project massively to the CA3 region via the perforant path [31].

The hippocampus proper projects its output (i) to the subiculum and the deep layers of the entorhinal cortex via the angular bundle, (ii) to several subcortical areas (e.g., the nucleus accumbens (NA)) via the fornix (FX). In particular, we consider the output of CA1 cells that reaches the nucleus accumbens via the fornix ${ }^{1}$. We identify the NA as the area where navigation control is achieved by means of reward-based learning $[2,32]$. We consider the dopaminergic input that NA receives from the ventral tegmental area (VTA). Indeed, dopamine neuron activity codes for external rewarding stimulation [35].

1 Actually, the fornix receives most of its inputs from the subiculum. However, experiments show that CA1 cells also project into it [31].

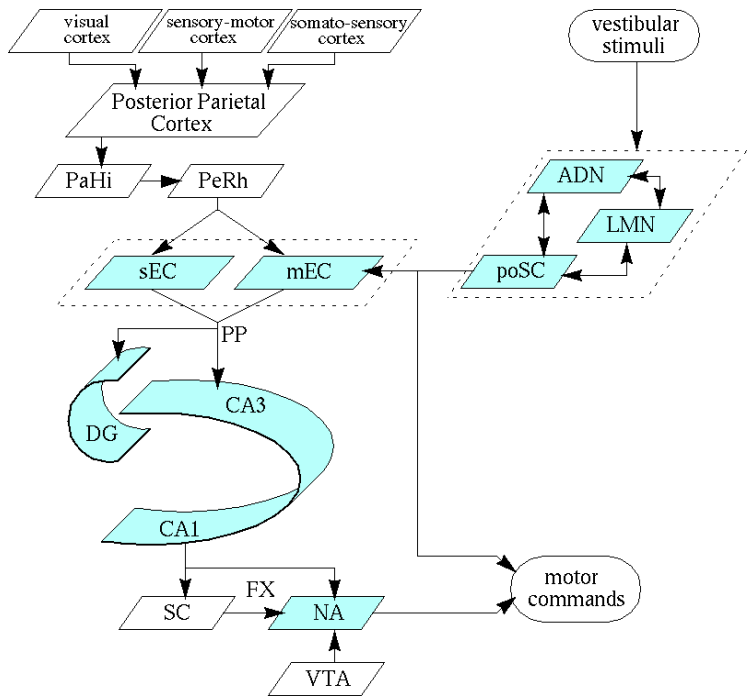

Fig. 2. A simplified overview of the anatomical counterparts of the constituents of our model. Glossary: PaHi: parahippocampal cortex, PeRh: perirhinal cortex, poSC: postsubiculum, LMN: lateral mammillary nuclei, $\mathrm{ADN}$ : anterodorsal nucleus of anterior thalamus (ATN), mEC: medial entorhinal cortex, sEC: superficial entorhinal cortex, DG: dentate gyrus, SC: subiculum, NA: nucleus accumbens, VTA: ventral tegmental area, PP: perforant path, FX: fornix. The hippocampus proper consists of the CA3-CA1 areas. The hippocampal formation consists of the hippocampus proper, the dentate gyrus, the entorhinal cortex, and the subicular complex $[31,3]$. Adapted from Redish and Touretzky [32], and from Burgess et al. [3].

\subsection{Learning Place Fields}

The model system consists of a multi-layer neural architecture that models high-dimensional continuous sensory input by means of overlapping place fields. Starting with no prior knowledge, the system grows incrementally and on-line as the agent interacts with the environment. Unsupervised Hebbian learning is used to detect the low-dimensional view manifold representing the visual input space. However, since distinct spatial locations might provide identical visual stimuli, such a view manifold might be singular [19]. Hebbian learning is applied to correlate visual cues and path integration in order to remove such singularities. The combination of internal and external stimuli yields a stable state space representation. On the one hand, unreliable visual data can be compensated for by means of path integration. On the other hand, reliable visual information can be used to reset the path integrator system.

\subsubsection{Representation of Visual Input}

We apply a simple computational strategy to emulate the feature-extraction mechanism observed in the visual cortex. Moving up the visual pathway, visual neurons become responsive to stimuli of increasing complexity, from orientation sensitive cells, to neurons sensitive to more complex patterns, such as faces [33].

We model spatio-temporal relationships between visual cues by means of neural activity. Incoming visual stimuli are interpreted by mapping images into a filteractivity space (Fig. 3). We define several classes of Walshlike filters ${ }^{2}$. Each class corresponds to a specific visual

${ }^{2}$ Walsh filters are simple and permit effective and low-cost feature-detection in one-dimensional visual spaces. We are cur- 


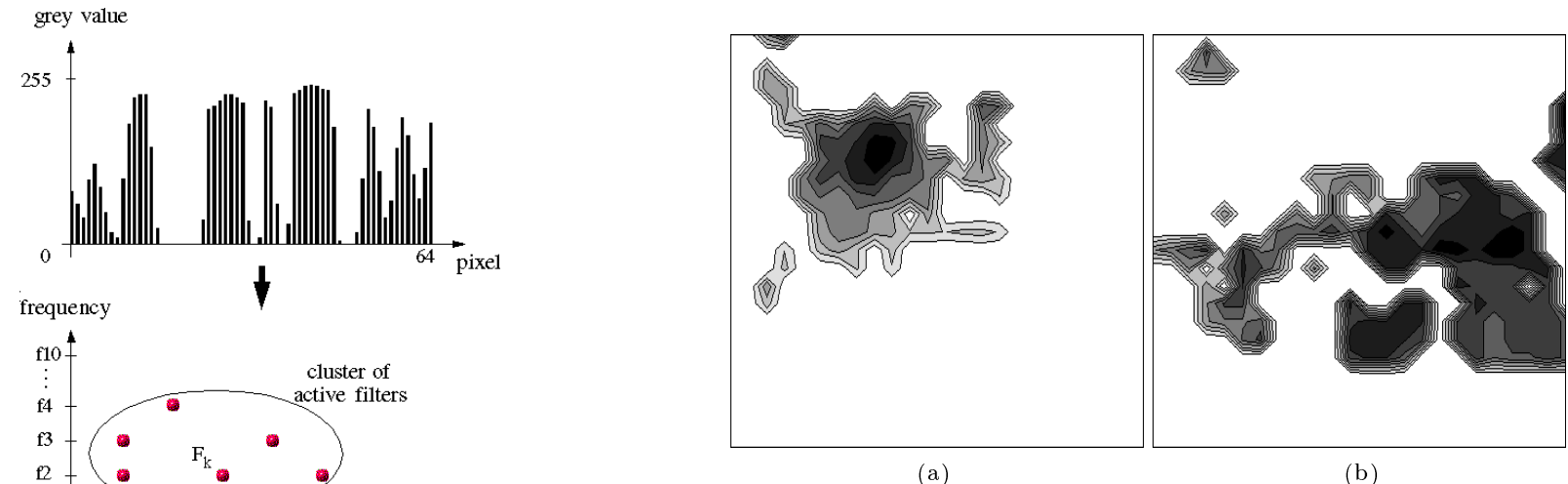

(a)

(b)

Fig. 4. Two examples of receptive fields of cells in our superficial entorhinal layer. The darker a region, the higher the firing rate of the cell when the robot is in that region of the environment. (a) The visual input is reliable, so that the maximal activity is confined to a localized spot in the environment. (b) The receptive field has multiple peaks indicating that similar visual stimuli occur at different locations.

Fig. 3. Linear images (top) are mapped into a filter activity space (bottom). Along the $\mathrm{x}$-axis we have different Walsh-like filters, $p_{1}, \ldots, p_{n}$, each of which responds to a specific pattern. Along the $y-$ axis the spatial frequency of each pattern $p_{i}$ is varied to represent the same pattern seen from different distances. Each image is encoded by the cluster of filters which maximally respond to that image.

pattern. The set of filters in that class corresponds to different spatial frequencies for that pattern (which endows the system with a distance discrimination property). In total we define 5 different classes of filters each containing filters at 10 different frequencies. Let $F_{k}$ be one our Walsh filters, where $1 \leq k \leq 50$ is the index of the filter, and let $l_{k}$ be its length (i.e., number of pixels covered by the filter). Given the input image $\mathbf{x}=\left(x_{1}, \ldots, x_{64}\right)$, the response $a_{k}$ of filter $F_{k}$ is computed by convolution

$a_{k}=\max _{n}\left\{\sum_{i=0}^{l_{k}-1} F_{k}(i) x_{n+i}\right\}$

where $0 \leq n \leq 64-l_{k}$. Since $-1 \leq x_{j} \leq 1$ and $F_{k}(i)=$ \pm 1 for all $i, k$, the relationship $\left|a_{k}\right| \leq l_{k}$ holds.

Each neural filter $F_{k}$ responds to a particular pattern. In order to detect more complex features, we consider a layer of visual cells one synapse downstream the neural filter layer. We call these neurons snapshot cells. The idea is to represent each image by the cluster of filters with the highest activation value, defined by Eq. 1. Let $C=0.7 \cdot l_{k}$ be the threshold above which a filter $F_{k}$ is considered as active. Given an image $\mathbf{x}$, the set of active filters projects one layer forward to form a snapshot cell

$s c=\left\{F_{k} \mid a_{k} \geq C\right\}$

The firing activity $r_{j}$ of a snapshot cell $s c_{j}$ is given by

$r_{j}=\frac{\sum_{k \in s c_{j}} \mathcal{H}\left(a_{k}-C\right)}{N_{j}}$

where $\sum_{k \in s c_{j}}$ sums over all the $N_{j}$ filters projecting to the cell $s c_{j}$, and $\mathcal{H}$ is the Heaviside function. The normalization has been chosen so that $0 \leq r_{j} \leq 1$.

\subsubsection{Allothetic Representation: Place Fields in the Superficial Entorhinal Cortex}

The activity of snapshot cells depends on the current gaze direction, and does not truly code for a spatial location. In order to achieve spatial sensitivity, we apply

rently implementing our model on a two-dimensional vision system by using biologically inspired Gabor filters [12].

unsupervised Hebbian learning to create a population of place cells one synapse downstream of the snapshot cell layer. We suppose that the anatomical counterpart for this neural layer is the superficial entorhinal cortex (Fig. 2). We call these neurons $s E C$ cells.

Every time the robot is at a new location, all simultaneously active snapshot cells are connected to a newly created sEC cell. Each new synapse is given a random weight in $(0,1)$. Let $i$ and $j$ be indices for sEC cells and snapshot cells, respectively. If $r_{j}$ is the firing activity of a snapshot cell $j$, then

$w_{i j}^{\text {new }}=\mathcal{H}\left(r_{j}-\epsilon\right) \operatorname{rnd}_{0,1}$

where $\epsilon=0.75$ is the activity threshold above which a snapshot cell is considered to be active. The firing rate $r_{i}$ of a sEC cell $i$ is given by the average activity $r_{j}$ of its presynaptic neurons $j$

$r_{i}=\frac{\sum_{j} w_{i j} r_{j}}{\sum_{j} w_{i j}}$

Once synapses are established, their efficacy is changed according to a Hebbian learning rule

$\Delta w_{i j}=r_{j}\left(r_{i}-w_{i j}\right)$

where $j$ is the index of the presynaptic neuron. If the robot is visiting a spatial location, it first checks whether there are already sEC cells coding for this location. New connections from snapshot cells to new sEC cells are created only if

$\sum_{i} \mathcal{H}\left(r_{i}-\epsilon\right)<A$

that is, only if the number of sEC cells activated at that location does not exceed a threshold $A$. Eq. 7 is a mere algorithmic implementation. We believe, however, that in some way rodents must have a possibility to detect novelty. Eq. 7 allows the system to control the redundancy level in the resulting spatial representation. We call the learning scheme defined by Eqs. 4, 6, and 7, an unsupervised growing network (see, e.g., [11]).

By definition, each sEC cell is driven by a set of snapshot cells whose activities code for visual features 


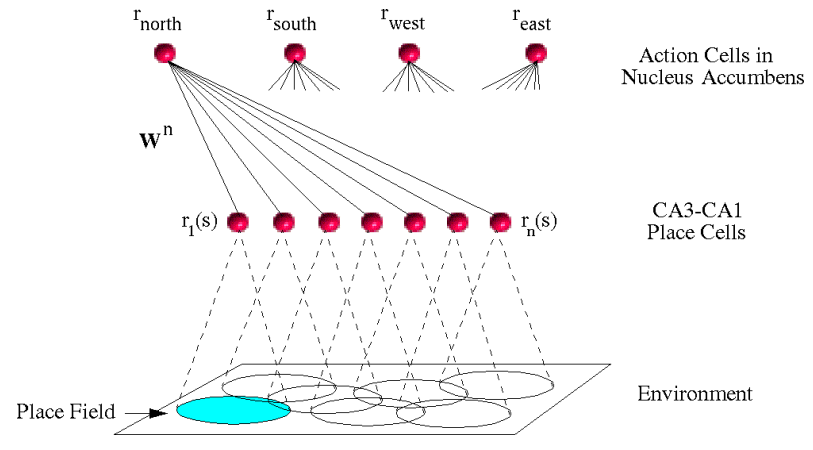

Fig. 10. CA3-CA1 place cells project to action cells (four for each target type) in the nucleus accumbens. Reinforcement learning is used to find the function which maps continuous spatial locations to locomotor actions.

formation (via the fornix) as well as rewarding stimulation from dopaminergic neurons (via the ventral tegmental area) [31].

We consider a population of action cells in the nucleus accumbens whose activity provides directional motor commands [2]. For each type of target (e.g., food or water), four action cells (coding for north, south, west, east allocentric actions) are driven by the population of CA3-CA1 place cells [4]. Synapses from hippocampal place cells to action cells are modified to learn the continuous location-to-action mapping function in goal-directed tasks. LTP occurs to associate spatial locations to rewarding actions, otherwise LTD takes place (Fig. 10).

Learning an action-value function over a continuous location space endows the system with spatial generalization capabilities. Thus, the robot may be able to associate appropriate actions to spatial positions that it has never seen before. Overlapping localized place fields in the CA3-CA1 layer, provide a natural set of basis functions that can be used to learn such a mapping function.

Let $\mathbf{s}$ be the robot's location (state), and let $a$ be an action cell in the nucleus accumbens, with $a \in \mathcal{A}:=$ $\{$ north, south, west, east $\}$. Let us denote the activation of a CA3-CA1 place cell $i$ by $r_{i}$, and the activity of an action cell $a$ by $r_{a}$. A robot position $\mathbf{s}$ is encoded by the place cell activity $\mathbf{r}(\mathbf{s})=\left(r_{1}(\mathbf{s}), r_{2}(\mathbf{s}), \ldots, r_{n}(\mathbf{s})\right)$, where $n$ is the number of CA3-CA1 place cells. Let $\mathbf{w}^{a}=\left(w_{1}^{a}, \ldots, w_{n}^{a}\right)$ be the synaptic projections from hippocampal place cells to the action cell $a$ (Fig. 10). The activity $r_{a}$ depends linearly on the robot's position $\mathbf{s}$ and on the synaptic weights $\mathbf{w}^{a}$ :

$r_{a}(\mathbf{s})=\left(\mathbf{w}^{a}\right)^{T} \mathbf{r}(\mathbf{s})=\sum_{i=1}^{n} w_{i}^{a} r_{i}(\mathbf{s})$

The learning task consists of updating $\mathbf{w}^{a}$ to approximate the optimal goal-oriented function which maps states $\mathbf{s}$ into action cell activity $r_{a}(\mathbf{s})$. To do this, we use the linear gradient-descent version of Watkins' Qlearning algorithm [37]. Given a robot position s, we interpret the neural activity $r_{a}(\mathbf{s})$ as the "expected gain" when taking action $a$ at location $\mathbf{s}$ of the environment.

During training, the robot behaves in order to either consolidate goal-directed paths (exploitation) or find novel routes (exploration). This exploitation-exploration trade-off is determined by an $\epsilon$-greedy action selection policy, with $0 \leq \epsilon \leq 1$ [37]. At each time $t$, the robot takes the "optimal" action $a_{t}^{*}$ with probability $1-\epsilon$ (exploitation)

$a_{t}^{*}=\arg \max _{a} r_{a}\left(\mathbf{s}_{t}\right)$

or, it might resort to uniform random action selection with probability equal to $\epsilon$ (exploration). At each time step $\Delta t$, the synaptic efficacy of projections $\mathbf{w}^{a}$ changes according to [37]

$\Delta \mathbf{w}^{a}=\alpha \delta_{t} \mathbf{e}_{t}$

The terms in Eq. 15 have the following interpretation:

(i) The factor $\alpha, 0 \leq \alpha \leq 1$, is a constant learning rate.

(ii) The term $\delta_{t}$ is the prediction error defined by

$\delta_{t}=R_{t+1}+\gamma \max _{a} r_{a}\left(\mathbf{s}_{t+1}\right)-r_{a}\left(\mathbf{s}_{t}\right)$

where $R_{t+1}$ is the actual reward delivered by an internal brain signal, and $\gamma, 0 \leq \gamma \leq 1$, is a constant discount factor. The temporal difference $\delta_{t}$ estimates the error between the expected and the actual reward when, given the location $\mathrm{s}$ at time $t$, the robot takes action $a$ and reaches location $\mathbf{s}^{\prime}$ at time $t+1$. Training trials allow the robot to minimize this error signal. Thus, asymptotically $\delta_{t} \approx 0$, which means that, given a state-action pair, the deviation between predicted and actual rewards tends to zero.

Neuro-physiological data show that the activity of dopamine neurons in mammalian midbrain encodes the difference between expected and actual occurrence of reward stimuli [35]. In particular, the more reliably a reward is predicted, the more silent a dopaminergic neuron. Thus, the temporal difference error $\delta_{t}$ used to update our synaptic weights $\mathbf{w}^{a}$ may be thought of as a dopamine-like teaching signal.

(iii) During training paths, Eq. 15 allows the robot to memorize action sequences. Since recently taken actions are more relevant than earlier ones, we need a memory trace mechanism to weight actions as a function of their occurrence time. The vector $\mathbf{e}_{t}$, called eligibility trace, provides such a mechanism [37]. The update of the eligibility trace depends on whether the robot selects an exploratory or an exploiting action. Specifically, the vector $\mathbf{e}_{t}$ changes according to

$\mathbf{e}_{t}=\mathbf{r}\left(\mathbf{s}_{t}\right)+ \begin{cases}\gamma \lambda \mathbf{e}_{t-1} & \text { if exploiting } \\ 0 & \text { if exploring }\end{cases}$

where $\lambda, 0 \leq \lambda \leq 1$, is a trace-decay parameter [37], and $\mathbf{r}\left(\mathbf{s}_{t}\right)$ is the CA3-CA1 vector activity. We start with $\mathbf{e}_{0}=\mathbf{0}$.

\subsection{Behavioral Experiments}

Given the experimental setup shown in Fig. 6, we define a specific target region (e.g., a feeding location) within the environment. We apply the above reward-based learning scheme to build up a navigational strategy leading the robot toward the target from any location, while avoiding obstacles. In this work, we do not address the problem of consolidating and recalling hippocampal representations [31]. We simply assume that entering a familiar environment results in recalling the hippocampal 
chart associated with this environment [21]. To study robot behavior, we adopt the same protocol as employed by neuro-ethologists with rats [31]. Navigational maps are learned through a training session consisting of a sequence of trials. Each trial begins at a random location and ends when the robot reaches the target. At the beginning of each trial the robot retrieves its starting location on the hippocampal chart based on the allothetic (visually-driven) representation (Sec. 2.2.2) [21, 20].

During learning we consider a discrete set of four actions $\mathcal{A}=\{$ north, south, west, east $\}$. However, after learning, population vector coding is applied to map $\mathcal{A}$ into a continuous action space $\mathcal{A}^{\prime}$ by averaging the ensemble action cell activity. Given a position $\mathbf{s}$ of the robot, the action $a^{\prime}(\mathbf{s}) \propto\left(\begin{array}{c}\cos \phi \\ \sin \phi\end{array}\right)$ is a direction in the environment encoded by the action cell activity in the nucleus accumbens

$a^{\prime}(\mathbf{s})=\frac{\sum_{a \in \mathcal{A}} a r_{a}(\mathbf{s})}{\sum_{a \in \mathcal{A}} r_{a}(\mathbf{s})}$

where $a_{n}=\left(\begin{array}{c}0 \\ 1\end{array}\right), a_{s}=\left(\begin{array}{c}0 \\ -1\end{array}\right), a_{w}=\left(\begin{array}{c}-1 \\ 0\end{array}\right)$, and $a_{e}=\left(\begin{array}{l}1 \\ 0\end{array}\right)$ are the four principal directions. Eq. 18 results in smooth trajectories.

The experiments have been carried out with a learning rate $\alpha=0.1$, a discount factor $\gamma=1.0$, and a decay factor $\lambda=0.9$. The reward-signal function $R(\mathbf{s})$ is defined by

$R(\mathbf{s})= \begin{cases}1 & \text { if } \mathbf{s}=\text { target state } \\ -0.5 & \text { if } \mathbf{s}=\text { collision state } \\ 0 & \text { otherwise }\end{cases}$

where collision means contact with walls or obstacles.

We adopt a dynamically changing $\epsilon$-probability. The idea is to increase the probability of exploring novel routes as the time to reach the target increases. The $\epsilon$ parameter is defined by the exponential function

$\epsilon(t)=\frac{\exp (\beta t)+k_{1}}{k_{2}}$

where $\beta=0.068, k_{1}=100$, and $k_{2}=1000$, where $t=0,1,2, \ldots$ are discrete time steps. If we consider the dynamic of $\epsilon$ over a time window of 100 time steps, at $t=0$ the robot behaves according to a value $\epsilon=0.101$ (i.e., enhancing exploitation), and at $t=100$ it behaves according to a value $\epsilon=1.0$ (i.e., enhancing exploration). If at the end of the time, $t=100$, the target is not reached yet, exploration is further enhanced by keeping a fixed $\epsilon=1.0$ for another 100 time steps. Then, exploitation is resumed by setting $t=0$ and $\epsilon=0.101$. Moreover, every time the target is reached the time window is re-initialized as well, and $\epsilon$ is set equal to 0.101 . These are heuristic methods to ensure a sufficient amount of exploration.

\subsubsection{Experiment with a single target type (e.g., food)}

Fig. 11(a) shows a two-dimensional view of the arena of Fig. 6(a). White objects are transparent obstacles. Only infrared sensors can detect obstacles, which are transparent with respect to the vision system. Since obstacles are

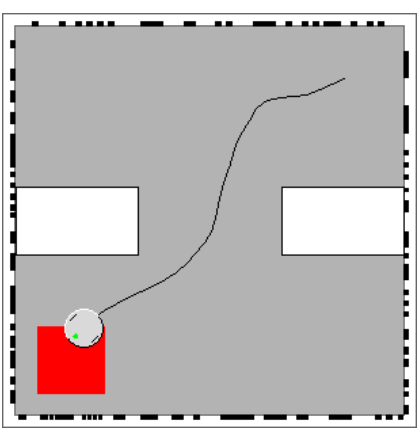

(a)

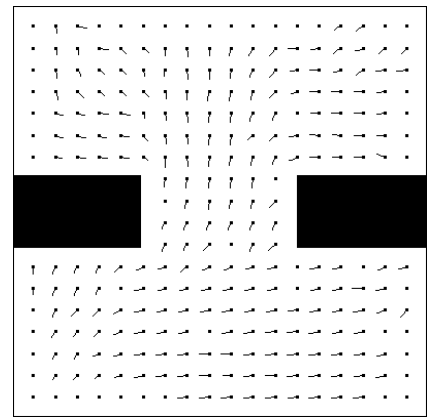

(b)
Fig. 11. (a) A two-dimensional view of the environment with a feeder location (dark grey square), and two obstacles (white rectangles). An example of robot trajectory induced by the action cell activity after learning. (b) Vector field representation of the learned navigational map.

not visible and have been added after learning, the place fields in the model are not affected. The dark square represents the feeder location. The target area is about 2.5 times the area occupied by the robot (grey circle). In Fig. 11(b) we show the navigational map learned by the robot in about 920 time steps, which correspond to 50 trials from random starting positions to the target. The vector field representation of Fig. 11(b) has been obtained by rastering uniformly over the whole environment. Dots represent sampled positions and pointers indicate the direction calculated from Eq. 18 at each position. Finally, the solid line shown in Fig. 11(a) is an example of a robot trajectory from a novel starting location using the learned navigational map.

\subsubsection{Moving the learned target}

This experiment consists of changing the location of a previously learned target, and of allowing the robot to adapt its navigational behavior consequently. The idea is to endow the system with an internal reward-expectation mechanism.

During training trials, the robot learns to correlate the CA3-CA1 place cell activity to the positive reward signal, $R=1$, which it receives at the food location. This is achieved by considering a neuron $d$, that we call the reward-expectation cell, one synapse downstream the place cell layer (Fig. 12(a)). Let $i$ be an index over the CA3-CA1 cell population. Connections $w_{d i}$ from place cells to the reward predicting cell are inhibitory synapses, and are initialized to random values within the interval $[-0.1,0]$. The cell $d$ receives as input the external rewarding stimulus $R$ as well. The activity $r_{d}$ of cell $d$ is non linear and it is defined by

$r_{d}= \begin{cases}f\left(\sum_{i} w_{d i} r_{i}\right)+R & \text { if } R \geq 0 \\ 0 & \text { otherwise. }\end{cases}$

where $f(x)=\tanh (x)$. Thus, the activity of cell $d$ depends on both the external reward $R$ and the CA3-CA1 network activity.

In order to learn the desired correlation between the event "positive reward" and the place cell activity, we apply Hebbian learning and modify the inhibitory weights $w_{d i}$ by an amount 


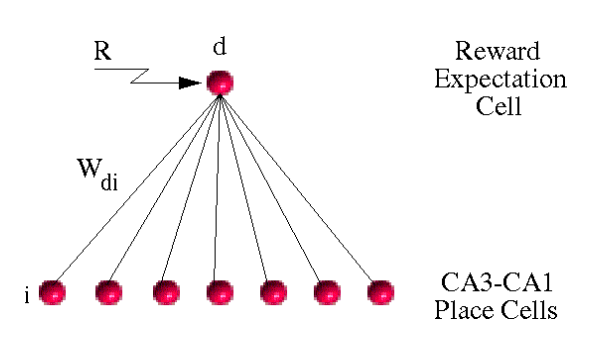

(a)

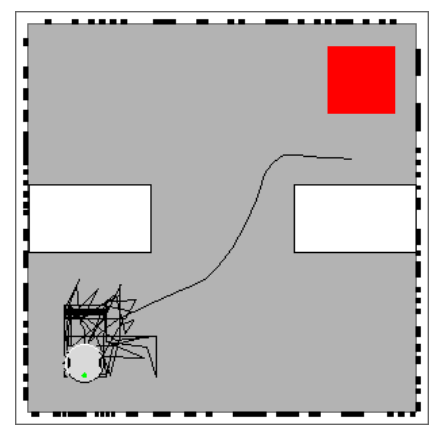

(b)

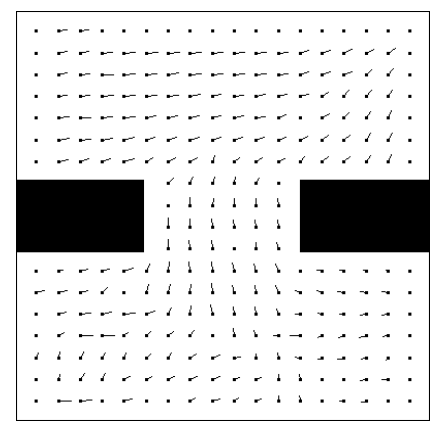

(c)
Fig. 12. (a) The internal reward-expectation mechanism. The activity of cell $d$ depends on the CA3-CA1 place cell activity and on the external reward signal $R$. (b) The arena and the previously learned target (dark square) which has been moved to a new location. Solid lines represent trajectories of the robot searching for the previously learned food location. (c) The re-adapted navigational map corresponding to the new rewarding location.

$\Delta w_{d i}=r_{i} r_{d}\left(w_{d i}-1\right)$

The more correlated the activity $r_{i} r_{d}$, the more inhibitory the synapses $w_{d i}$.

As a consequence, before correlating the external reward signal with internal spatial representation, cell $d$ responds maximally when the robot receives a positive $R=1$. Indeed, since weights $w_{d i}$ are initially close to zero, the activity $r_{d} \approx R=1$ (according to Eq. 21). As training proceeds, the robot starts predicting the external stimulus $R$ by learning synapses $w_{d i}$. Then, every time the robot is near the target location, the cell $d$ receives a strong inhibitory input $\sum_{i} w_{d i} r_{i}$ which compensates for the excitatory reward $R$. Thus, when $R$ is fully predicted, even if the robot receives the $R=1$ signal the cell $d$ remains silent. On the other hand, if the fully predicted reward signal fails to occur (i.e., the learned target has been moved away), the activity of cell $d$ is strongly depressed $\left(r_{d} \approx-1\right)$, and an internal negative reward is generated. When the number of collected negative internal rewards exceeds a fixed threshold $D$ (e.g., $D=10$ ), the robot "forgets" the previous target location and starts looking for a new goal. Fig. 12(b) shows the same environment of Fig. 11(a) where the previously learned target has been moved to another location. The robot is attracted by the previous feeder position and it accumulates internal negative rewards. Fig. 12(c) presents the navigational map re-adapted to the new food location.

Our reward-expectation cell $d$ finds its neuro-physiological counterpart in dopaminergic neurons observed in mammalian midbrain. The response of these neurons is a function of the unpredictability of incoming stimuli [35]. In particular, they respond positively to external rewards which occur unpredictably. Instead, they remain silent if a fully predicted stimulus arrives. By contrast, when a fully expected reward fails to occur, dopamine neurons respond negatively exactly at the time at which the reward is expected [35]. Instead of Eq. 21, we could have also used the prediction error $\delta_{t}$ defined in Eq. 16 to monitor an unexpected target location.

\subsubsection{Experiment with multiple target types (e.g., food and water)}

The reward-based learning scheme described in Sec. 3.1, Fig. 10, can also be applied to multiple target types. Let $\mathbf{T}=\left\{T_{1}, \ldots, T_{m}\right\}$ be a set of distinct target types (e.g., $T_{1}$ could be a food location, $T_{2}$ a water location, and so on). For each given target $T_{i}$ we consider a set of location-to-action mapping function $r_{a}^{T_{i}}(\mathbf{s})$, and a set of synaptic weights $\mathbf{w}^{a, T_{i}}$. We also consider distinct rewarding signals $\mathbf{R}=\left\{R^{T_{1}}, \ldots, R^{T_{m}}\right\}$. Then, we adopt the above Q-learning algorithm to approximate the $r_{a}^{T_{i}}(\mathbf{s})$ functions.

In this experiment we consider two distinct types of rewarding stimulations $T_{1}$ (food) and $T_{2}$ (water). Fig. 13(a) shows the two target locations (left and right bottom squares) within the environment. The learning session starts by focusing on the feeder location $T_{1}$. Thus the primary task for the robot is to approximate the $r_{a}^{T_{1}}(\mathbf{s})$ functions. The navigational map learned during about 1300 time steps is shown in Fig. 13(b).

Notice that when searching for food it might happen that the robot encounters the water location and receives a positive reward signal with respect to $T_{2}, R^{T_{2}}=1$. This information can be exploited by the robot by adjusting $\mathbf{w}^{T_{2}}$ weights. That is, even if $T_{2}$ is not the current target, the robot can partially learn a navigational map leading to it. Fig. 13(c) shows the knowledge about the water location $T_{2}$ acquired by the robot while learning the optimal policy to reach the food $T_{1}$. Thus, when the robot decides to focus on the water target (i.e., to approximate the $r_{a}^{T_{2}}$ (s) action cell activity), it does not start from zero knowledge. This results in a shorter learning time for $T_{2}$, and accelerates the robot's progress. Fig. 13(d) presents the navigational map learned by the robot after about 440 time steps when looking for water.

\section{Discussion}

We have presented a computational model of the hippocampus to study its role in spatial cognition and navigation. Even if it relies on neuro-physiological experimental data, the proposed neural architecture is highly simplified with respect to biological hippocampal circuitry.

In particular, we have stressed the importance of integrating external and internal stimuli to drive place cell activity in CA3-CA1 regions [28, 32]. An allothetic vision-based representation is formed in a model of the superficial entorhinal cortex. Spatial properties of the environment are extracted from visual inputs in order to 


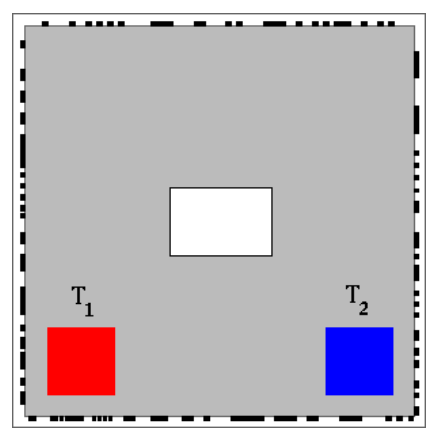

(a)

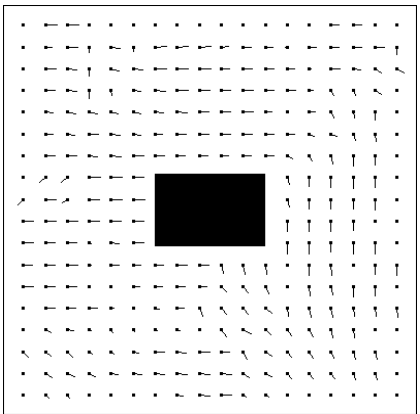

(c)

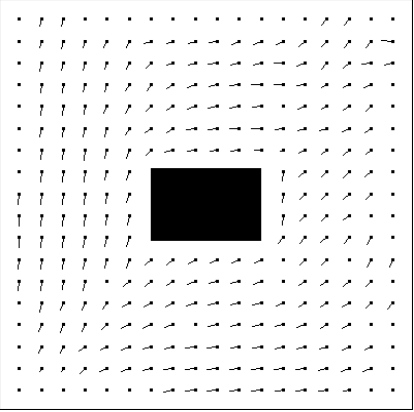

(b)

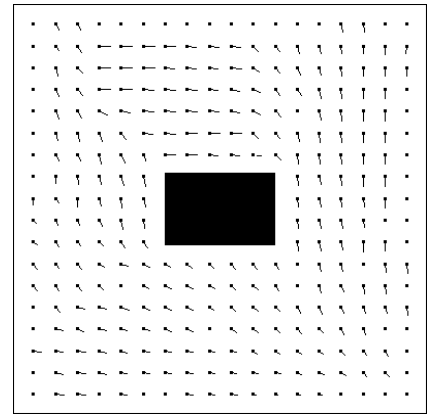

(d)
Fig. 13. (a) The arena with two distinct target types $T_{1}$ (e.g., food) and $T_{2}$ (e.g., water). The white rectangle is an obstacle. (b) The navigation map corresponding to the food rewarding location $T_{1}$. (c) The partial navigation map corresponding to the water location $T_{2}$ learned by the robot when focusing on food $T_{1}$. (d) The final map acquired by the robot when focusing on water $T_{2}$.

characterize distinct regions of the environment by combinations of visual cues. On the other hand, an idiothetic representation takes place in our model of the medial entorhinal cortex, integrating the internal movementrelated information provided by proprioception. Allothetic and idiothetic representations converge onto CA3CA1 areas of the hippocampus and form a rather stable place fields representation. Allothetic and idiothetic charts are correlated by associative learning. This induces a mutual benefit in the sense that path integration may disambiguate visual singularities [9] and, conversely, visual information may be used for resetting the path integration [21]. This process is done on-line during the development of the hippocampal space representation (i.e., exploration). A threshold mechanism is used to evaluate the reliability of the visual input being used for dead-reckoning calibration.

Unsupervised Hebbian learning is applied to build the neural system incrementally and on-line. Redundancy in the place cell activity is considered as a crucial property to yield robustness. After learning, the model has developed a spatial representation consisting of a large population of overlapping place fields covering the environment uniformly and densely. To interpret the ensemble place cell activity as spatial locations we apply population vector coding $[14,41]$.

The hippocampus projects to the nucleus accumbens, a subcortical structure involved in spatial behavior $[2,31]$. We consider a population of locomotor action neurons [4] in the nucleus accumbens and we apply reward-based learning to adjust synapses from CA3-CA1 cells to action cells [2]. For a given target location, this results in learning a mapping function from the continuous space of physical locations to the activity space of action cells. This allows us to accomplish goal-oriented navigation based on the neural activity in the nucleus accumbens. Navigation maps are derived by interpreting the ensemble action cell activity by means of population coding [4]. Note, however, that while population vector decoding allows us an interpretation of the place cell activity, this interpretation is not necessary for action learning by reinforcement: For Q-learning, place cells are simply a set of basis functions in the high-dimensional input space. Burgess et al. [4] have previously postulated a goal-memory system consisting of a population of goal cells $(G C)$ driven by hippocampal place cells. The goal cell activity encodes the animal's position with respect to the goal (i.e., north, east, south, west). In his model, however, only the activity of hippocampal cells whose place field contains the target is correlated to the $\mathrm{GC}$ activity by Hebbian learning. This results in GC of limited attraction radius which impairs the animal's navigation at large distances from the target and does not allow for detours around obstacles. In addition, Burgess et al. [4] do not propose any re-learning mechanism to cope with targets whose location might change.

A robotic platform has been used to validate our computational model in real task-environment contexts. There is, of course, a whole body of work on robot navigation with neural networks (e.g., $[9,27,7]$ ), but only few authors have previously implemented hippocampal models on real robots $[4,13,19]$. Understanding the underlying mechanisms of hippocampal place cell activity offers the attractive prospect of developing control algorithms that directly emulate mammalian navigational abilities. On the other hand, the simplicity and the transparency of artificial agents make them suitable for studying and understanding neuro-physiological processes.

In the future, data analysis will be focused on the dynamics of the robot behavior using the same methodology as employed by ethologists for living animals. In particular, we will evaluate our hippocampal model through experiments concerning environment manipulations (e.g., shrinking and stretching the arena, changing light conditions). We are interested in studying the potential conflicts which might occur between allothetic and idiothetic information [10], and in modeling the mutual relationships between path integration and visual stimuli. For example, a system which is dominated by vision-based information will show stretched place fields in a stretched environment, whereas a system which mainly relies on path integration will not. Hopefully, a systematic study of these effects will allow us to make neuro-ethological predictions concerning animals trained in controlled environments [10].

\section{Acknowledgments}

Supported by the Swiss National Science Foundation, project nr. 21-49174.96. The authors thank Dario Floreano for useful discussions. 


\section{References}

1. H. Blair and P. Sharp. Anticipatory head direction signals in anterior thalamus: Evidence for a thalamocortical circuit that integrates angular head motion to compute head direction. $J$. of Neuroscience, 15(9):6260-6270, 1995.

2. M. Brown and P. Sharp. Simulation of spatial-learning in the Morris water maze by a neural network model of the hippocampal-formation and nucleus accumbens. Hippocampus, 5:171-188, 1995.

3. N. Burgess, K. Jeffery, and J. O'Keefe. Integrating hippocampal and parietal functions: A spatial point of view. In K. J. N. Burgess and J. O'Keefe, editors, The Hippocampal and Parietal Foundations of Spatial Cognition, chapter 1, pages 3-29. Oxford University Press, 1999.

4. N. Burgess, M. Recce, and J. O'Keefe. A model of hippocampal function. Neural Networks, 7:1065-1081, 1994.

5. T. Collett and J. Zeil. Places and landmarks: An arthropod perspective. In S. Healy, editor, Spatial Representation in Animals, chapter 2, pages 18-53. Oxford University Press, 1998.

6. P. Dayan. Navigating through temporal difference. In R. Lippmann, J. Moody, and D. Touretzky, editors, Neural Information Processing Systems 3, pages 464-470. Morgan Kaufmann, San Mateo, CA, 1991.

7. J. del R. Millán. Rapid, safe, and incremental learning of navigation strategies. IEEE Trans. on Systems, Man and Cybernetics-Part B, 26:408-420, 1996.

8. J. Droulez and A. Berthoz. The concept of dynamic memory in sensorimotor control. In D. Humphrey and H.-J. Freund, editors, Motor Control: Concepts and Issues, pages 137-161. John Wiley Sons, 1991.

9. T. Duckett and U. Nehmzow. Mobile robot self-localization and measurement of performance in middle scale environments. J. of Robotics and Autonomous Systems, 24(1-2):5769, 1998.

10. A. Etienne, J. Berlie, J. Georgakopoulos, and R. Maurer. Role of dead reckoning in navigation. In S. Healy, editor, Spatial Representation in Animals, chapter 3, pages 54-68. Oxford University Press, 1998.

11. B. Fritzke. Growing cell structures -A self-organizing network for unsupervised and supervised learning. Neural Networks, 7(9):1441-1460, 1994.

12. D. Gabor. Theory of communication. J. of the IEE, 93:429457, 1946 .

13. P. Gaussier, C. Joulain, A. Revel, S. Zrehen, and J. Banquet. Building grounded symbols for localization using motivation. In Fourth European Conf. on Artificial Life, pages 299-308, 1997.

14. A. Georgopoulos, A. Schwartz, and R. Kettner. Neuronal population coding of movement direction. Science, 233:1416-1419, 1986.

15. A. Hill and P. Best. Effects of deafness and blindness on the spatial correlates of hippocampal unit activity in the rat. Exp. Neurology, 74:204-217, 1981.

16. M. Jung and B. McNaughton. Spatial selectivity of unit activity in the hippocampal granular layer. Hippocampus, 3(2):165182, 1993.

17. J. Knierim, H. Kudrimoti, and B. McNaughton. Place cells, head direction cells, and the learning of landmark stability. $J$. of Neuroscience, 15:1648-1659, 1995

18. C. Leonhard, R. Stackman, and J. Taube. Head direction cells recorded from the lateral mammillary nuclei in rats. Soc. for Neuroscience Abstr., 22:1873, 1996.

19. H. Mallot, M. Franz, B. Schölkopf, and H. Bülthoff. The viewgraph approach to visual navigation and spatial memory. In W. Gerstner, A. Germond, M. Hasler, and J. Nicoud, editors, Artificial Neural Networks - ICANN'97. 7th Int. Conf., pages 751-756, Lausanne, Switzerland, 1997. Springer Verlag.
20. B. McNaughton. Neural mechanisms for spatial computation and information storage. In L. Nadel, L. Cooper, P. Culicover, and R. Harnish, editors, Neural Connections, Mental Computation, chapter 9, pages 285-350. MIT Press, Cambridge, MA, 1989.

21. B. McNaughton, C. Barnes, J. Gerrard, K. Gothard, M. Jung, J. Knierim, H. Kudrimoti, Y. Qin, W. Skaggs, M. Suster, and K. Weaver. Deciphering the hippocampal polyglot: the hippocampus as a path integration system. J. of Experimental Biology, 199:173-185, 1996.

22. B. McNaughton, C. Barnes, and J. O'Keefe. The contributions of position, direction, and velocity to single unit activity in the hippocampus of freely-moving rats. Exp. Brain Research, $52: 41-49,1983$

23. R. Muller, E. Bostock, J. Taube, and J. Kubie. On the directional ring properties of hippocampal place cells. J. of Neuroscience, 14(12):7235-7251, 1994.

24. J. O'Keefe and J. Dostrovsky. The hippocampus as a spatial map: Preliminary evidence from unit activity in the freely moving rat. Brain Research, 34:171-175, 1971.

25. J. O'Keefe and L. Nadel. The Hippocampus as a cognitive map. Clarendon Press, Oxford, 1978.

26. J. O'Keefe and M. Recce. Phase relationship between hippocampal place units and the EEG theta rhythm. Hippocampus, 3:317-330, 1993.

27. R. Pfeifer and C. Scheier. Understanding Intelligence. MIT Press, Cambridge, MA, 1999.

28. G. Quirk, R. Muller, and J. Kubie. The firing of hippocampal place cells in the dark depends on the rat's recent experience. J. of Neuroscience, 10(6):2008-2017, 1990.

29. G. Quirk, R. Muller, J. Kubie, and J. Ranck. The positional firing properties of medial entorhinal neurons: Description and comparison with hippocampal place cells. J. of Neuroscience, 12(5):1945-1963, 1992.

30. M. Recce, A. Speakman, and J. O'Keefe. Place fields of single hippocampal cells are smaller and more spatially localized than you thought. Soc. of Neuroscience Abstr., 17:484, 1991.

31. A. Redish. Beyond the cognitive map. PhD thesis, Dep. of Computer Science, Carnegie Mellon University, Pittsburgh, PA, 1997.

32. A. Redish and D. Touretzky. Cognitive maps beyond the hippocampus. Hippocampus, 7(1):15-35, 1997.

33. E. Rolls and M. Tovée. Sparseness of the neuronal representation of stimuli in the primate temporal visual cortex. $J$. of Neurophysiology, 73:713-726, 1995.

34. E. Salinas and L. Abbott. Vector reconstruction from firing rates. J. of Comp. Science, 1:89-107, 1994.

35. W. Schultz, P. Dayan, and R. Montague. A neural substrate of prediction and reward. Science, 275:1593-1599, 1997.

36. P. Sharp and C. Green. Spatial correlates of firing patterns of single cells in the subiculum of freely moving rat. $J$. of Neuroscience, 14(4):2339-2356, 1994.

37. R. Sutton and A. Barto. Reinforcement learning, an introduction. MIT Press-Bradford Books, Cambridge, Massachusetts, 1998.

38. J. Taube. Place cells recorded in the parasubiculum of freely moving rats. Hippocampus, 5(6):569-583, 1996.

39. J. Taube, R. Muller, and J. Ranck. Head direction cells recorded from the postsubiculum in freely moving rats. I. Description and quantitative analysis. J. of Neuroscience, 10:420-435, 1990.

40. O. Trullier and J. Meyer. Place sequence learning for navigation. In W. Gerstner, A. Germond, M. Hasler, and J. Nicoud, editors, Artificial Neural Networks - ICANN'97. 7th Int. Conf., pages 757-762, Lausanne, Switzerland, 1997. Springer Verlag.

41. M. Wilson and B. McNaughton. Dynamics of the hippocampal ensemble code for space. Science, 261:1055-1058, 1993.

This article was processed by the author using the $\mathrm{LAT}_{\mathrm{E}} \mathrm{X}$ style file cljour2 from Springer-Verlag. 